\title{
Reflections on FDA Draft Guidance for Products Containing Nanomaterials: Is the Abbreviated New Drug Application (ANDA) a Suitable Pathway for Nanomedicines?
}

\author{
Marden Emily, ${ }^{1}$ Ntai Ioanna, ${ }^{2}$ Bass Scott, ${ }^{3}$ and Flïhmann Beat ${ }^{2,4}$
}

Received 1 June 2018; accepted 12 August 2018; published online 20 August 2018

\begin{abstract}
$\begin{array}{lll}\text { Abstract. } & \text { The US Food and Drug Administration (FDA) recently released a draft }\end{array}$ guidance for industry titled "Drug Products, Including Biological Products, that Contain Nanomaterials." The FDA's attention to the unique safety and efficacy aspects of drugs containing nanomaterials is commendable. This Draft Guidance succeeds in acknowledging the complexity of these products, as well as the challenges associated with approving safe and therapeutically equivalent complex generic versions. However, the challenge posed by the manufacturing process for drugs containing nanomaterials is insufficiently addressed. The critical quality attributes of such products cannot be properly defined, and therefore it is not possible to design informative comparative physicochemical assessments for equivalence. As a consequence, the 505(j) Abbreviated New Drug Application (ANDA) pathway, currently advised as the standard from the FDA, is not suitable for the approval of complex generic products. Drawing from the successful story of biologics, we propose instead a stepwise totality-of-evidence approach, demonstrating similarity and including clinical studies when deemed necessary, as an appropriate alternative to the 505(j) ANDA pathway.
\end{abstract}

KEY WORDS: complex generic; FDA; nanomedicines; nanosimilar; non-biological complex drugs (NBCDs).

The US Food and Drug Administration (FDA or Agency) recently released a draft guidance for industry titled "Drug Products, Including Biological Products, that Contain Nanomaterials" (1). The FDA's issuance of this guidance signals a welcome focus on the unique safety and efficacy aspects of this expanding category of therapeutic products. The Draft Guidance stands as a significant milestone in acknowledging the complexity of drug products containing nanomaterials, as well as the challenges associated with approving complex generics that ensure patient safety.

Despite its recognition of significant challenges in approving complex generics, the FDA seems to suggest that nanomedicine generics can be approved via the standard Abbreviated New Drug Application (ANDA) approval pathway through Section 505(j) of the Food, Drug, and Cosmetic Act (FDCA). Section 505(j) was developed for drug products where the drug substance is a small molecule. Drug products that contain nanomaterials can also contain drug

\footnotetext{
${ }^{1}$ Food, Drug and Medical Device Regulatory Law, Sidley Austin LLP, Palo Alto, California, USA.

${ }^{2}$ Non-Biological Complex Drugs, Vifor Pharma Ltd., Flughofstrasse 61, 8152, Opfikon, Switzerland.

${ }^{3}$ Global Life Sciences, Sidley Austin LLP, Washington, D.C., USA.

${ }^{4}$ To whom correspondence should be addressed. (e-mail: beat.fluehmann@viforpharma.com)
}

substances that are small molecules. Critically, the active pharmaceutical ingredient has been intentionally altered in such a way that its new nano-scale size has given it novel properties, resulting in modified behaviors in the body with a different safety and efficacy profile (e.g., changed solubility/ bioavailability for nanocrystals). Ultimately, this leads to the question of whether the whole drug product should be considered as the active pharmaceutical ingredient (API), making it more complex than a non-complex small-moleculecontaining drug product (2). Such an approach is supported by both the United States Pharmacopeia and the European Pharmacopoeia, who both already define the whole entity of certain nanomedicines as the drug product, in their respective monographs. For example, iron sucrose is referred to in both monographs as iron sucrose solution, rather than as simply iron sucrose $(3,4)$.

Herein, we outline the FDA's Draft Guidance and suggest that the Agency reconsider the approval pathway for complex generic products. The Draft Guidance covers a large variety of drug products and many different routes of administration, ranging from oral to topical to parenteral. For precision, we have chosen to comment from the scope of parenteral iron products, since complex generics of originator products have already been approved in many countries, and there is a plethora of evidence revealing safety and efficacy differences between them and their originator. In addition, IV 
products have been characterized as high risk and are likely to "exhibit clinically significant changes in exposure, safety, and/or effectiveness relative to the referenced product" (1). Although these products are singled out in such a way, we would suggest that it might be a wider concern linked to the nature of the nanoparticles contained in nanomedicines.

\section{FDA DRAFT GUIDANCE ON DRUG PRODUCTS CONTAINING NANOMATERIALS ACKNOWLEDGES COMPLEXITY OF CATEGORY}

In the Agency's description, the category of drug products containing nanomaterials includes those:

1. having "at least one external dimension, or an internal or surface structure, in the nanoscale range (approximately $1 \mathrm{~nm}$ to $100 \mathrm{~nm}$ )" or

2. exhibiting "properties or phenomena, including physical or chemical properties or biological effects, that are attributable to its dimension (s), even if these dimensions fall outside the nanoscale range, up to one micrometer $(1,000 \mathrm{~nm}) "(1)$.

The FDA goes on to identify the unique considerations applicable to the development and approval of safe and effective drug products containing nanomaterials. Toward this end, the FDA lists 11 factors that it identifies as crucial to making a risk-based evaluation of these drug products. These are:

- Adequacy of characterization of the material structure and its function

- Complexity of the material structure

- Understanding of the mechanism by which the physicochemical properties of the material impact its biological effects (e.g., effect of particle size on pharmacokinetic parameters)

- Understanding the in vivo release mechanism based on the material physicochemical properties

- Predictability of in vivo release based upon established in vitro release methods

- $\quad$ Physical and chemical stability

- Maturity of the nanotechnology (including manufacturing and analytical methods)

- Potential impact of manufacturing changes, including in-process controls and the robustness of the control strategy on critical quality attributes of the drug product

- Physical state of the material upon administration

- $\quad$ Route of administration

- Dissolution, bioavailability, distribution, biodegradation, accumulation, and their predictability based on physicochemical parameters and animal studies (1)

The Draft Guidance makes clear that the critical quality attributes (CQAs) of a drug product containing nanomaterials must incorporate these factors to the extent they "potentially impact the quality, safety, or efficacy" of the product (1). According to the Agency, determining the safety and efficacy of such products requires a risk-based approach, which entails "continual reduction of residual uncertainty through a product's lifecycle" (1).
Given these challenges, most drug products containing nanomaterials could also be described as "non-biological complex drugs" (NBCDs). The scientific community has developed this term to describe drug products in which the active substance is not a homo-molecular structure-as is the case for non-complex small-molecule drug products-but, like biological products, consists of different closely related and often nano-particulate structures that cannot be isolated and fully quantitated, characterized, and/or described by physicochemical analytical means $(5,6)$. As such, NBCDs present a challenge to the regulatory concept of pharmaceutical equivalence, which is central to the approval of generic copies. While not all NBCDs contain structures strictly within the nanoscale range of 1 to $100 \mathrm{~nm}$, the composition, quality, and in vivo performance of NBCDs are highly dependent on the manufacturing process (5-8).

Despite acknowledging the complexity of drug products containing nanomaterials, the Draft Guidance seems to suggest that the 505(j) ANDA pathway is appropriate for all complex generic versions. Under the 505(j) pathway, a generic approval relies on the finding that the complex generic drug product is bioequivalent to the reference listed drug product (RLD) [FDCA $\S \S 505(j)(2)$ and (4)].

In the Draft Guidance, the FDA even seems to extend the availability of the 505(j) pathway to parenteral products containing nanomaterials, though it acknowledges that such products are at "high risk to exhibit clinically significant changes in exposure, safety, and/or effectiveness relative to the referenced product" (1). To address such issues, the FDA states that:

the applicant should demonstrate the generic product contains the same active and inactive ingredients as the RLD (i.e. is qualitatively [Q1] the same) in the same concentration (i.e. is quantitatively [Q2] the same) as the RLD. In general, the generic applicant should conduct in vivo bioequivalence (BE) studies, demonstrate comparable size and distribution of nanomaterials based on population $\mathrm{BE}$ criteria, and demonstrate sameness in a wide range of physicochemical properties (1).

The FDA goes on to articulate the significant challenges to meeting these requirements for a parenteral drug product containing nanomaterials. These issues include:

1. the active ingredients in such drug products are "heterogeneous mixtures which may require considerable characterization to demonstrate drug substance sameness";

2. the "manufacturing processes for certain nanomaterials are complicated and involve lengthy steps";

3. the "drug substance often exists in multiple forms, e.g., free drug or nanomaterial-associated drug, both in systemic circulation and at the target site"; and

4. "drug levels in systemic circulation may not always reflect drug concentration at the target site" (1).

Taking these challenges into account, the FDA acknowledges that "evidence of comparable pharmacokinetic (PK) parameters in blood/plasma in conventional BE studies alone may not be sufficient to satisfy the requirements for generic drug approval" (1). Ultimately, the FDA advises the ANDA 
applicant to undertake additional measures such as comparative physicochemical testing to "enable an accurate assessment of the PK of the generic product" (1).

In fact, the challenges in demonstrating Q1 and Q2 sameness and bioequivalence of nanomaterial-containing drug products have been demonstrated to be far more profound. First, as explained in a recent publication coauthored by industry scientists, academic researchers, and regulators (including the FDA), the science is not advanced enough to adequately identify relevant CQAs that must be assessed to ensure the safety of a generic (7). Most nanomaterial-containing drug products do not consist of a homo-molecular pharmaceutical moiety, as multiple parts of the drug directly affect the treatment outcome. The Draft Guidance acknowledges that "the active ingredients of some nanomaterials are generally heterogeneous mixtures which may require considerable characterization to demonstrate drug substance sameness" (1). The scientific community and other stakeholders agree that such products are comprised of different closely related structures and there is not a single substance to be isolated, quantitated, and fully characterized, but they acknowledge that characterization is still elusive, even by state-of-the-art physicochemical analytical means (8). Such products cannot be adequately characterized to support a determination of sameness and will differ as a result of differences in manufacturing process. In addition, these products have different pharmacokinetic and pharmacodynamic properties, as a result of their varying physicochemical characteristics that cannot be determined at the same level of detail as for materials or end products that do not have at least one dimension in the nanoscale or are not engineered to exhibit properties or phenomena attributable to their dimension (as the application of nanotechnology is described in the Draft Guidance) (1). The net result is that it may not be possible to adequately characterize drug products containing nanomaterials, and therefore sameness simply cannot be demonstrated. Taking that into consideration and scrutinizing the term complex generic, which implies therapeutic equivalence and substitutability, it is worth considering whether an alternative term should be used, one that describes accurately and more appropriately the nature of those products, which are not identical but, rather, similar, to the originator nanomedicine.

\section{COMPLEXITY OF PRODUCTS MEANS THAT THE 505(J) ANDA PATHWAY CANNOT ADEQUATELY ENSURE SAFETY AND EFFICACY}

Given the challenges described in the previous section, it would make sense for the FDA to take a more nuanced approach to establishing the safety and efficacy of a complex generic product. For example, the Agency could apply a stepwise approach, such as the one used for biosimilars. Rather than relying solely on physicochemical equivalence, it should only be the first step in a longer path that could include in vivo, animal, and clinical studies, to secure the necessary evidence to demonstrate biosimilarity. This would enable the FDA to require those studies that are necessary to ensure the safety and efficacy of complex generic drug products containing nanomaterials prior to administering them to patients.
The challenge posed by the manufacturing process for drug products containing nanomaterials is insufficiently addressed in the Draft Guidance. The FDA notes in passing that "any critical structural change in the multiple components of nanomaterial-based products can influence the bioequivalence, pharmacodynamics and toxicology profiles" of nanomaterials (1). In fact, there is evidence that even slight differences between the manufacturing process for a nanomaterial-containing drug product and a complex generic product can significantly impact efficacy or safety, and have adverse effects on patients. A recent paper demonstrated how important the manufacturing process is, by comparing different batches of the iron sucrose originator product to different batches of several iron sucrose complex generics (9). Not only were there differences in hydrodynamic size range between the originator product and the complex generics, but higher inter-batch variability was observed for the complex generics, which was linked to lack of robustness in the manufacturing process by the authors (9). Andre Raw, senior scientific and policy advisor at FDA's Office of Life Cycle Products, said during a symposium on the equivalence of complex drug products that "understanding the nature of the complexity of these drugs (referencing glatiramer acetate and enoxaparin sodium originator products) is key to evaluating generic similars" (10). Departing from specific examples, he stressed the overall importance of the manufacturing process by saying that "... equivalence in these (manufacturing process) signatures ensures that the generic active ingredient is manufactured with effectively the same polymerization kinetic and cleavage biases as the brand name."

In the USA, the FDA has only approved one complex generic for an originator parenteral iron product via the 505(j) ANDA pathway, which is the complex generic sodium ferric gluconate (SFG) complex in sucrose. Following the approval in 2011, two years later the FDA published a product-specific guidance for SFG IV products (11). Very recently, a study looking at the physicochemical profile of the originator ferumoxytol injection iron product, compared this profile to both the originator and generic SFG products (12). In doing so, it revealed lower iron accumulation in $\mathrm{T}$ cells for the generic SFG product, compared to the originator SFG product (12). Whether this difference could have an impact on patients is unknown, as no clinical study is available. Therefore, we turn to look at additional examples of approved iron complex generics in the EU, the results of which are a clear indication that there is still a lot that is not known and more studies are required.

One study evaluated the effects of switching iron treatment in 75 consecutively stable, hemodialysis-dependent, chronic kidney disease patients, from the nanomaterialcontaining drug product iron sucrose originator product to a complex generic iron sucrose product (iron sucrose similar-ISS) that has been deemed to have met standards of therapeutic equivalence by French regulators (13). After the switch, hemoglobin levels in patients decreased rapidly and anemia medication had to be increased to return to targeted hemoglobin levels (13). In addition, there was a decrease in corresponding transferrin saturation (TSAT) and serum ferritin values after patients were switched from the iron sucrose originator to the complex generic product (13) indicating non-equivalence of the therapy. 
These findings regarding marked differences in efficacy between an original iron sucrose formulation and an approved ISS have been affirmed by a prospective and multicenter study in a much larger cohort (14). There, anemia parameters, and doses of erythropoietin stimulating agents (ESAs) and of iron sucrose, were prospectively recorded before and after the switch in 342 patients with a follow-up period of 56 weeks (14). Though the hemoglobin levels were stable in the study patients, the mean dose of IV iron sucrose per patient necessary to achieve appropriate levels decreased by $34.3 \%$ after switching from the complex generic iron sucrose to the original iron sucrose product (14). The mean dose of ESA decreased by $12.5 \%$ per patient over the same period after the switch to the original iron sucrose drug product (14).

Meanwhile, significant positive effects were also seen on mean TSAT levels and serum ferritin levels following the switch from the complex generic to the original iron sucrose product (14). Over the same period, the mean TSAT level increased by $6.8 \%$ and sodium ferritin levels rose by $12.4 \%$ (14).

Other complex generic iron sucrose products have been associated with an elevated risk of adverse events, even where the complex generic products had been evaluated and approved as therapeutically equivalent to the RLD. More specifically, a retrospective study looking back at data from 658 patients treated between September 2004 and December 2011 with either original iron sucrose or an ISS showed more frequent adverse drug reactions in the ISS (15). These events were mild to moderate in nature and were predominately injection site reactions and phlebitis (15).

These studies support the idea that initial comparable physicochemical profiles do not necessarily translate in the same safety and efficacy profile in patients. The FDA should revisit its suggestion that comparative physicochemical testing is sufficient to support generic approvals of drug products containing nanomaterials, given that the mechanisms of action by which these products impact biological targets within the body are not fully understood. The FDA presumes that comparable structural features will ensure compatible tissue distribution, when instead a totality-of-evidence approach that includes biological, preclinical, and clinical data evaluated in a stepwise manner is more appropriate for the complexity of these products $(16,17)$. However, the requested factors outlined by the FDA, namely:

- understanding of the mechanism by which the physicochemical properties of the material impact its biological effects (e.g., effect of particle size on pharmacokinetic parameters) and

- understanding the in vivo release mechanism based on the material physicochemical properties

are not met for numerous iron carbohydrates. The CQAs for such products cannot be properly defined, and therefore it is not possible to design informative comparative physicochemical assessments. In a seeming acknowledgement of this challenge, FDA initiated a clinical study comparing the safety and efficacy of brand and generic intravenous sodium ferric gluconate complexes, after it had already approved the generic version based on physicochemical testing, the results of which are still pending $(16,18)$. It will be interesting to see how it will compare to the results of a pilot study, which evaluated the physicochemical profiles of the brand and generic versions, and saw both similarities and differences in the range of methods used (16).

\section{FDA SHOULD ADOPT A STEPWISE APPROACH TO APPROVAL OF COMPLEX GENERIC VERSIONS OF DRUG PRODUCTS CONTAINING NANOMATERIALS}

Ultimately, the 505(j) ANDA pathway is not adequate to approve safe and effective complex generic versions of all drug products containing nanomaterials. In particular, nanomaterial-containing drug products (1) cannot be adequately characterized to support a determination of sameness, (2) will differ as a result of differences in manufacturing process, and (3) have different pharmacokinetic and pharmacodynamic properties as a result of their varying physicochemical characteristics that cannot be determined at the same level of detail as for non-complex small-molecule drug products. In his remarks at the July 18, 2017, meeting on the Hatch-Waxman Amendments, Commissioner Gottlieb recognized this reality, stating:

in many cases, the traditional requirements used to demonstrate sameness may not be appropriate when it comes to complex drugs that can't be easily measured in the blood, or where the drug's therapeutic affect is delivered locally to a particular organ, rather than systemically, through the blood (19).

The Draft Guidance implicitly acknowledges this point, noting that "drug products containing targeted nanomaterials intended for systemic action and that are administered intravenously, are likely to present high risk" (1).

Forcing potential complex generic versions of such drug products into the 505(j) ANDA pathway, without directly addressing these known risks through clinical studies (20), means that "generic" versions may be approved that are not as safe or effective, as the reference products $(6,21,22)$. This practice is not in the best interest of patients. In respect of generic versions of intravenous iron-based nano-colloidal products, the European Medicines Agency has stated that, if the results of quality, non-clinical, or human PK studies show minor differences between the two products, a therapeutic equivalence study might be necessary to address their impact on safety and efficacy (21). In addition, the FDA research staff has noted that a better understanding of physicochemical characterization techniques is needed for iron carbohydrate colloid drug products (22).

A stepwise approach to approval of complex generic drug products containing nanomaterials would allow the FDA to have all information necessary to ensure safety and efficacy. In the next iteration of the Guidance, the FDA could take the position that it will model approval requirements for the category of complex generic drug products containing nanomaterials, on the biosimilars pathway. According to the FDA's relevant biosimilars guidance documents, structural and functional characterization form the first step and the foundation of the informational requirements necessary to demonstrate biosimilarity. In this context, the FDA recommends relying on a fingerprint-like analysis that can cover a large number of product attributions and their combinations 
with high sensitivity (23). Then, depending on the "residual uncertainty" remaining after consideration of the results of these analytical studies, the FDA determines the scope and extent of additional studies necessary to demonstrate biosimilarity - such as animal studies, PK studies, pharmacodynamic (PD) studies, immunogenicity studies, and comparative clinical trials-in a "stepwise manner" (24). This approach allows the uncertainty regarding the impact of minor differences on safety and efficacy to be resolved by increasingly higher order testing performed on a step-by-step basis, to ultimately demonstrate that the biosimilar product will have the same clinical outcome in all patients. In this sense, the biosimilars approval pathway gives the FDA great flexibility, including authority to require more, or less, clinical evidence as the Agency determines appropriate in order to ensure patient safety. Unlike in the world of Shakespeare, where a rose would still smell sweet even if it were called something else, names in regulatory science should demonstrate precision, as "complex generics" implies strict therapeutic equivalence, which is unattainable in the case of drug products containing nanomaterials. Rather, the biosimilarinspired term "nanosimilar" is more appropriate and takes into consideration the nature of nanomaterial-containing products $(25,26)$.

\section{CONCLUSION}

The FDA's acknowledgement of the complex issues raised by drug products containing nanomaterials, particularly as greater numbers of such drug products are being developed and introduced to the marketplace, is an important step in the right direction. In light of this complexity, the FDA's suggested reliance on the standard FDCA 505(j) ANDA pathway for complex generic products seems illadvised. Indeed, the FDA already recognizes in the Draft Guidance that drug products containing nanomaterials pose significant risks to patients, and that the standard Q1 and Q2 approach may not adequately demonstrate safety and efficacy. It is hoped that the FDA will revise the text of its Draft Guidance to specify that for such products, approval of complex generics should follow a stepwise approach, including clinical studies, when necessary. This change should also be reflected on the terminology used; thus, the more precise term "nanosimilar" is suggested.

Open Access This article is distributed under the terms of the Creative Commons Attribution 4.0 International License (http://creativecommons.org/licenses/by/4.0/), which permits unrestricted use, distribution, and reproduction in any medium, provided you give appropriate credit to the original author(s) and the source, provide a link to the Creative Commons license, and indicate if changes were made.

\section{REFERENCES}

1. U.S. FDA. Draft guidance for industry: drug products, including biological products, that contain nanomaterials. December 2017. https://www.fda.gov/downloads/Drugs/ GuidanceComplianceRegulatoryInformation/Guidances/ UCM588857.pdf. Accessed 9 Apr 2018.
2. Registrar Corp. U.S. FDA Drug Definitions https:// www.registrarcorp.com/fda-drugs/definitions/. Accessed 16 Jul 2018.

3. U.S. Pharmacopoeia. USP monographs: iron sucrose injection. http://www.pharmacopeia.cn/v29240/usp29nf24s0_m42475.html. Accessed 16 Jul 2018.

4. European Pharmacopoeia. Monograph number 02753: iron hydroxide sucrose concentrated solution. https:// extranet.edqm.eu/4DLink1/4DCGI/Web_View/mono/2753. Accessed 16 Jul 2018.

5. Lygature. Non-Biological Complex Drugs (NBCD) Working Group. https://www.lygature.org/non-biological-complex-drugsworking-group. Accessed 9 Apr 2018.

6. Schellekens H, Stegemann S, Weinstein V, de Vlieger JS, Flühmann B, Mühlebach S, et al. How to regulate nonbiological complex drugs (NBCD) and their follow-on versions: points to consider. AAPS J. 2014;16(1):15-21. https://doi.org/10.1208/ s12248-013-9533-z.

7. Hussaarts L, Mühlebach S, Shah VP, McNeil S, Borchard G, Flühmann B, et al. Equivalence of complex drug products: advances in and challenges for current regulatory frameworks. Ann N Y Acad Sci. 2017;1407(1):39-49. https://doi.org/10.1111/ nyas.13347.

8. Flühmann B, de Vlieger JSB, Vulto AG, Mühlebach S, Weinstein V, Shah VP. The authorization of non-biological complex drugs (NBCDs) follow-on versions: specific regulatory and interchangeability rules ahead? GaBI Journal. 2013;2(4):204-7. https://doi.org/10.5639/gabij.2013.0204.054.

9. Di Francesco T, Philipp E, Borchard G. Iron sucrose: assessing the similarity between the originator drug and its intended copies. Ann N Y Acad Sci. 2017 Nov;1407(1):63-74. https:// doi.org/10.1111/nyas.13517.

10. The New York Academy of Sciences. Equivalence of complex drug products: scientific and regulatory challenges. Academy eBriefings. 2016. www.nyas.org/ComplexDrugs16-eB. Accessed $16 \mathrm{Jul} 2018$.

11. U.S. FDA. Draft guidance on sodium ferric gluconate complex. June 2013. https://www.fda.gov/downloads/Drugs/ GuidanceComplianceRegulatoryInformation/Guidances/ UCM358142.pdf. Accessed 16 Jul 2018.

12. Shah A, Mankus CI, Vermilya AM, Soheilian F, Clogston JD, Dobrovolskaia MA. Feraheme ${ }^{\circledR}$ suppresses immune function of human $\mathrm{T}$ lymphocytes through mitochondrial damage and mitoROS production. Toxicol Appl Pharmacol. 2018;350:5263. https://doi.org/10.1016/j.taap.2018.04.028.

13. Rottembourg J, Kadri A, Leonard E, Dansaert A, Lafuma A. Do two intravenous iron sucrose preparations have the same efficacy? Nephrol Dial Transplant. 2011;26(10):3262-7. https:// doi.org/10.1093/ndt/gfr024.

14. Agüera ML, Martin-Malo A, Alvarez-Lara MA, GarciaMontemayor VE, Canton P, Soriano S, et al. Efficiency of original versus generic intravenous iron formulations in patients on haemodialysis. PLoS One. 2015;10(8):e0135967. https:// doi.org/10.1371/journal.pone.0135967.

15. Lee ES, Park BR, Kim JS, Choi GY, Lee JJ, Lee IS. Comparison of adverse event profile of intravenous iron sucrose and iron sucrose similar in postpartum and gynecologic operative patients. Curr Med Res Opin. 2013;29(2):141-7. https://doi.org/10.1185/03007995.2012.760444.

16. Sun D, Rouse R, Patel V, Wu Y, Zheng J, Karmakar A, et al. Comparative evaluation of U.S. brand and generic intravenous sodium ferric gluconate complex in sucrose injection: physicochemical characterization. Nanomaterials. 2018;8(1) https:// doi.org/10.3390/nano8010025.

17. Mühlebach S. Regulatory challenges of nanomedicines and their follow-on versions: a generic or similar approach? Adv Drug Deliv Rev. 2018 Jun 30. pii: S0169-409X(18)30163-7. doi: https:// doi.org/10.1016/j.addr.2018.06.024.

18. U.S. FDA. Therapeutic equivalences of generic iron complex product. Solicitation No. FDA-SS-1116099. April 19, 2013. https:// www.fbo.gov/utils/view?id=220604efb0ab5d4c5b9e0d0e2be617a1. Accessed 9 Apr 2018.

19. U.S. FDA. Gottlieb S. Opening remarks for Part 15 Public Meeting on Generic Drug Competition. July 18, 2017. https://www.fda.gov/ NewsEvents/Speeches/ucm567323.htm. Accessed 9 Apr 2018. 
20. The United States Code. Federal Food, Drug, and Cosmetic Act (FDCA) § 505(j)(2)(A). http://uscode.house.gov/ view.xhtml? req=granuleid:USC-prelim-title21section355\&num $=0 \&$ edition=prelim. Accessed 9 Apr 2018.

21. EMA. EMA/CHMP/SWP/620008/2012: Reflection paper on the data requirements for intravenous iron-based nano-colloidal products developed with reference to an innovator medicinal product. March 26, 2015. http://www.ema.europa.eu/docs/ en_GB/document_library/Scientific_guideline/2015/03/ WC̄500184922.pdf. Accessed 9 Apr 2018.

22. Zou P, Tyner K, Raw A, Lee S. Physicochemical characterization of iron carbohydrate colloid drug products. AAPS J. 2017;19(5):1359-76. https://doi.org/10.1208/s12248-017-0126-0.

23. U.S. FDA. Guidance for industry: quality considerations in demonstrating biosimilarity of a therapeutic protein product to a reference product. April 2015. https://www.fda.gov/downloads/ drugs/guidances/ucm291134.pdf. Accessed 9 Apr 2018.
24. U.S. FDA. Guidance for industry: scientific considerations in demonstrating biosimilarity to a reference product: guidance for industry. April 2015. https://www.fda.gov/downloads/Drugs/ GuidanceComplianceRegulatoryInformation/Guidances/ UCM291128.pdf. Accessed 9 Apr 2018.

25. Ehmann F, Sakai-Kato K, Duncan R, Hernán Pérez de la Ossa D, Pita R, Vidal JM, et al. Next-generation nanomedicines and nanosimilars: EU regulators' initiatives relating to the development and evaluation of nanomedicines. Nanomedicine (Lond). 2013;8(5):849-56. https://doi.org/10.2217/nnm.13.68.

26. Mühlebach S, Borchard G, Yildiz S. Regulatory challenges and approaches to characterize nanomedicines and their follow-on similars. Nanomedicine (Lond). 2015 Mar;10(4):659-74. https:// doi.org/10.2217/nnm.14.189. 